\title{
PATTERNS OF SUBSTANCE ABUSE IN SCHIZOPHRENIA: NATURE AND SIGNIFICANCE
}

\author{
John R. DeQuardo, Christopher F. Carpenter and Rajiv Tandon \\ Schizophrenia Program, University of Michigan Medical Center, Ann Arbor, MI 48109-01 16, U.S.A.
}

(Received for publication 28 March 1994)

\begin{abstract}
Summary-Recent literature suggests that the increasingly prevalent problem of substance abuse may have important implications for the symptoms and course of schizophrenia. To further examine the impact of substance abuse on this disorder, the clinical and research charts of 67 schizophrenic patients admitted to the Schizophrenia Program of the University of Michigan between 1987 and 1990 were reviewed and data on symptomatology, history of substance use, age at onset and first hospitalization, and family psychiatric history were gleaned. This information was analyzed with respect to clinical, demographic, and outcome variables with the following findings: (i) female subjects abused substances at a much lower rate $(20 \%)$ than male subjects $(48 \%)$; (ii) substanceabusing patients with schizophrenia had lower pre- and post-treatment Hamilton Rating Seale of Depression scores, poorer post-discharge treatment compliance, and were younger at first hospitalization than were non-abusing patients; (iii) the most often abused substance was cannabis (28\%) followed closely by alcohol $(2.1 \%)$, with cocaine, hallucinogens, and stimulants all abused hy significantly fewer patients; (iv) rates of family history of schizophrenia were similar in substanceabusing and non-substance-abusing schizophrenic patients. These data suggest that substance abuse may not be etiologically related to schizophrenia but influences the onset. course, and symptomatology of schizophrenia.
\end{abstract}

\section{Introduction}

THE INCREASING prevalence of substance abuse may have important implications for the onset, clinical manifestations, course, and outcome of schizophrenia. This assertion is supported by the high frequency of patients dually-diagnosed with schizophrenia and substance abuse being encountered (Regier et al., 1990), and the growing number of papers appearing in the literature.

Although there is inherent difficulty in finding a causative role for substance abuse in schizophrenia due to the complex nature of the illness, many researchers have found a correlation between substance use and onset of illness. The amount of cannabis used by individuals has been cited as a risk factor for development of schizophrenia (Andreasson et al., 1987, 1989), as evidenced by the finding of an earlier onset of schizophrenia in cannabis-abusing patients (Andreasson et al., 1989; Breakey et al., 1974; Mueser et al., 1990) and chronic drug-abusing patients when compared to non-abusing schizophrenic patients (Tsuang et al., 1982). Production of a schizophreniform psychosis in previously healthy people by a single large dose of a dopaminergic agent (e.g. cocaine or amphetamine)

Address correspondence to: Dr DeQuardo, Schizophrenia Program, University of Michigan Medical Center, Ann Arbor, MI 48109-0116, U.S.A. 
(Weller et al., 1988) lends further support to the hypothesis that abuse of certain substances may cause schizophrenia. Alternative explanations for the association include increased prevalence of psychiatric disorders in the families of substance-abusing schizophrenics when compared to non-substance-abusing patients (i.e. genetic susceptibility, stress, learning) (Breakey et al., 1974), mistaking an organic psychosis for schizophrenia (Johnson et al., 1988), the possibility that schizophrenia and substance abuse are associated due to underlying personality structure (Johnson et al., 1988), and that substance abuse might be caused by an emerging schizophrenia (i.e. self-medication) (Andreasson et al., 1987). The idea that patients may self-medicate with drugs of abuse (and thereby alter symptoms) lends credence to the idea that substance abuse may alter the course and phenomenology of schizophrenia.

A recent review of studies examining substance abuse in schizophrenia found that patients tend to use more amphetamines, cocaine, cannabis, and hallucinogens, and less alcohol than do other psychiatric patients or normal controls (Schneier \& Siris, 1987). Numerous studies have reported that substance abuse poses a significant risk for schizophrenic patients, with possible relapse (Richard et al., 1985) or exacerbation of symptoms (Test et al., 1989; Treffert, 1978; Weller et al., 1988). There are also data suggesting an antagonistic cannabis neuroleptic interaction (Knudsen \& Vilmar, 1984) which could interfere with medication response and produce a poor outcome. Heavy substance abusers believe their use to be selfmedicating, whereas psychiatric staff note symptomatic exacerbation with use, together likely to contribute to poorer compliance and thus poorer outcome (Test et al., 1989). These findings are countered by results which suggest that cannabis abuse in schizophrenics correlates with fewer symptoms and a decreased number of hospitalizations (Mueser et al., 1990)

Studies focusing on the effect of substance abuse on outcome in schizophrenia generally suggest poorer outcome in substance-abusing patients. Specifically, Miller and Tanenbaum (1989) found that schizophrenic patients who abuse substances before admission have a significantly higher rate of A.M.A. discharges. Other studies have related substance abuse to poorer short-term outcome (Bowers et al., 1990; Safer, 1987); reports focusing on the longer term outcome of substance-abusing schizophrenic patients are few but generally suggest less favorable prognosis (Mueser et al., 1990). However, two recent reports dispute this negative impact on outcome in schizophrenia (Dixon et al., 1991; Zisook et al., 1992). Clearly there are discrepancies in the exact role substance abuse plays in the course and phenomenology of schizophrenia. A thorough study of the temporal relationship between substance abuse and schizophrenic symptoms could answer some of these questions; however, the methodology, in terms of controls and ethics, would be quite difficult to implement.

To examine these issues, we conducted a retrospective study of substance abuse in schizophrenia, focusing on pattern of abuse, family history, and clinical and demographic variables.

\section{Methodology}

The subjects were 67 consecutive schizophrenic patients admitted to the Schizophrenia Program at the University of Michigan between 1987 and 1990 for treatment of an 
exacerbation of their illness. Patients met both DSM III-R (American Psychiatric Association, 1987) and RDC (Feighner et al., 1972) criteria for schizophrenia based on admission evaluation by an experienced faculty psychiatrist. Positive, negative, and depressive symptoms were assessed using the Brief Psychiatric Rating Scale (BPRS) (Overall \& Gorham, 1962) "THOT" factor (sum of conceptual disorganization, suspiciousness, hallucinatory behavior and unusual thought content items), Scale for the Assessment of Negative Symptoms (SANS) (Andreasen, 1981), and the 17 item Hamilton Rating Scale of Depression (HRSD) (Hamilton, 1960) respectively, by a psychiatrist blind to the substance-abuse history of each patient; assessments were conducted at baseline (at least 2 weeks medication-free) and 4 weeks after clinically determined antipsychotic medication treatment; the same rater (R.T.) performed all symptom ratings. The patients' clinical and research charts were reviewed by a researcher blind to each patient's clinical characteristics for information on history of substance abuse, urine toxicology screen results, and blood alcohol level on admission. Demographic data included sex, race, age at admission, age at onset (first symptoms), age at first hospitalization, educational achievement, and family history of psychiatric illness (first and second degree relatives). This information was gathered by the admitting clinician and treatment team social worker from patient and family interviews and from review of all available prior records, which is part of the standard admission evaluation. One year outcome data, quantified via the StrausCarpenter Multidimensional Outcome Scale (Straus \& Carpenter, 1972), included number of hospitalizations, employment status, social activity, symptoms, global functioning, treatment compliance, and overall ratings. This instrument is an anchored scale that was scored from patient and family interviews completed by clinicians and social workers in the Schizophrenia Program. Urine toxicology results and/or blood alcohol data were available on 52 patients. Samples were initially screened via a radioimmunoassay technique the results of which were confirmed by gas chromatography-mass spectroscopy; specific cut-off values for a positive test are available on request from the authors.

For the purpose of this study, a substance abuser was defined as a heavy user of one or more substances; occasional (insignificant) users included those patients with limited past use of the substances assessed. Estimates of pattern of use and number of substances used were based on the assessment made by the psychiatrist performing the standard admission work-up; these are estimates of lifetime abuse status. The chart information was not of sufficient detail to permit diagnosis of specific DSM III-R substance-dependence syndromes, however, all patients in the abuse groups met DSM III-R criteria for substance abuse. It was also not possible to determine the timing of onset of substance abuse and rate of change of substance use from the chart data. Subjects were categorized into two mutually exclusive groups: Group 1 included abusers of alcohol, cannabis, cocaine, hallucinogens, and/or stimulants; Group 2 included all non-abusers, as well as those with substance use that was considered occasional; non-users and occasional users differed only in terms of sex ratio, and thus were combined for analysis.

Unpaired, two-tailed $t$-tests and the $\chi^{2}$ statistic were used to compare substance-abusing to non-substance-abusing schizophrenic patients on continuous and discrete variables, respectively. 


\section{Results}

Of the 67 subjects in this study, $25(37 \%)$ were female and $42(63 \%)$ male. There were $56(84 \%)$ white patients, $8(12 \%)$ African-Americans, and $3(5 \%)$ Asian-Americans. The mean age $( \pm S D)$ on admission for all patients was $28.7 \pm 8.6$ (range 17-54), for males $27.9 \pm 8.7$ (range 17-54), and for females $30.0 \pm 8.4$ (range 18-47). Among the female subjects, five $(20 \%)$ reported substance abuse (i.e. heavy use of at least one substance) and $20(48 \%)$ of the males reported substance abuse $\left(\chi^{2}=4.0, d f=1, p<.05\right)$. Overall, 25 $(37 \%)$ of subjects reported substance abuse. Male and female subjects did not differ in the number of substances abused. There were no differences in rates of substance use between racial groups. Of all substance abusers, $76 \%$ abused cannabis, $56 \%$ alcohol, $16 \%$ hallucinogens, $12 \%$ cocaine, $8 \%$ amphetamines, and $4 \%$ barbiturates; the percentages do not equal 100 due to the high frequency of polysubstance abuse. Subdivision of pattern of use of individual substances by sex and number of substances used by patients are displayed in Tables 1 and 2 .

Urine toxicology and/or blood alcohol levels were available on 52 of 67 patients $(77.6 \%)$; of these, nine were positive $(17.3 \%)$. Substances detected included cannabis, alcohol, benzodiazepines, and cocaine metabolites. Eight of the nine patients with positive results were from the substance-abusing group. This result is highly significant $\left(\chi^{2}=7.5, d f=1\right.$, $p<.01$ ), and validates the original grouping scheme.

Table 3 lists the results of two-tailed $t$-tests of the clinical symptom data. Sample mean values are as follows: pretreatment BPRS "THOT" $15.2 \pm 2.9$, post-treatment BPRS "THOT" $9.7 \pm 2.9$, pretreatment SANS $12.2 \pm 4.3$, post-treatment SANS $8.5 \pm 3.6$, pre-

Table 1

Percentage of Patients Abusing Individual Substances

\begin{tabular}{lccc}
\hline Abuse category & $M$ & $F$ & Total \\
\hline None & $22(32.8 \%)$ & $20(29.9 \%)$ & $42(62.7 \%)$ \\
Cannabis & $16(23.8 \%)$ & $3(4.5 \%)$ & $19(28.4 \%)$ \\
Alcohol & $11(16.4 \%)$ & $3(4.5 \%)$ & $14(21.0 \%)$ \\
Hallucinogens & $2(3.0 \%)$ & $2(3.0 \%)$ & $4(6.0 \%)$ \\
Cocaine & $2(3.0 \%)$ & $1(1.5 \%)$ & $3(4.5 \%)$ \\
Stimulants & $1(1.5 \%)$ & $1(1.5 \%)$ & $2(3.0 \%)$ \\
Barbiturates & 0 & $1(1.5 \%)$ & $1(1.5 \%)$ \\
\hline
\end{tabular}

Abuse totals do not equal number of subjects due to polysubstance use.

Percentages are of all subjects in the study.

Table 2

Percentage of Patients Engaged in Poly-substance Abuse

\begin{tabular}{lcccc}
\hline & \multicolumn{4}{c}{ Number of substances abused } \\
& 0 & 1 & 2 & 3 \\
\hline Male & $22(32.8 \%)$ & $10(14.9 \%)$ & $8(11.9 \%)$ & $2(3.0 \%)$ \\
Fcmale & $20(29.9 \%)$ & $1(1.5 \%)$ & $2(3.0 \%)$ & $2(3.0 \%)$ \\
Total & $42(62.7 \%)$ & $11(16.4 \%)$ & $10(14.9 \%)$ & $4(6.0 \%)$ \\
\hline
\end{tabular}

Percentages are of all subjects in the study. 
Table 3

Patient Symptom Data

\begin{tabular}{lrrrc}
\hline Clinical variable & $\begin{array}{c}\text { Abusers } \\
\text { Mean }+ \text { SD }\end{array}$ & $\begin{array}{c}\text { Non-abusers } \\
\text { Mean }+\mathrm{SD}\end{array}$ & $d f$ & $p$ \\
\hline Pre-HRSD & $11.4 \pm 2.6$ & $14.5 \pm 4.3$ & 65 & .01 \\
Post-HRSD & $6.6 \pm 2.7$ & $8.3 \pm 3.6$ & 64 & .05 \\
Change HRSD & $4.8 \pm 3.1$ & $6.0 \pm 2.8$ & 64 & $\mathrm{~ns}$ \\
Pre-BPRS ("THOT") & $15.1 \pm 2.6$ & $15.3 \pm 3.2$ & 65 & $\mathrm{~ns}$ \\
Post-BPRS ("THOT") & $9.1 \pm 2.2$ & $10.1 \pm 3.2$ & 65 & $\mathrm{~ns}$ \\
Change BPRS ("THOT") & $6.0 \pm 3.6$ & $5.2 \pm 3.0$ & 65 & $\mathrm{~ns}$ \\
Pre-SANS & $12.8+3.7$ & $11.8+4.6$ & 65 & $\mathrm{~ns}$ \\
Post-SANS & $8.4 \pm 3.0$ & $8.5 \pm 3.9$ & 65 & $\mathrm{~ns}$ \\
Change SANS & $4.5 \pm 3.1$ & $3.3 \pm 2.7$ & 65 & $\mathrm{~ns}$ \\
\hline
\end{tabular}

treatment HRSD $13.3 \pm 4.0$, post-treatment HRSD 7.7 \pm 3.4 . Patients in the substanceabusing group were rated as displaying fewer depressive symptoms (lower mean HRSD scores) both before and after treatment than patients with insignificant use. There were no significant differences between groups on pre- and post-treatment positive or negative symptoms or any index of symptom improvement after 4 weeks of clinically determined antipsychotic treatment.

The substance-abusing group's age at first hospitalization $( \pm S D)$ was $23.5 \pm 4.9$, for non-abusers it was $27.1 \pm 7.9$; mean age at first hospitalization for all patients was $25.7 \pm 7.1$ (range 15-47). Age at first hospitalization was lower in all substance-abusing patients compared to non-abusing patients $(t=2.0, d f=65, p<.05)$. The mean age $( \pm S D)$ at onset of symptoms for substance-abusing patients was $21.4 \pm 6.1$, for non-abusing patients was $23.2 \pm 6.4$, and for all patients was $22.5 \pm 6.3$ (range $7-41$ ). There was no difference in age at symptom onset between substance abusers and non-abusers. No other relationships between demographic variables and substance abuse status were found.

Examination of the family history data was without positive findings. There were no differences in rate of schizophrenia, alcohol abuse, or mood disorders in first or second degree relatives of substance abusing and non-abusing patients. Seven of 34 patients ( $21 \%$ ) had a family history of schizophrenia, 19 of $56(34 \%)$ had a family history of alcohol abuse, and 10 of 34 patients $(29 \%)$ had a family history of mood disorder.

Mean ratings of treatment compliance was also significantly different for substanceabusing patients and non-abusers. For substance abusers, the treatment compliance rating $( \pm S D)$ was $2.8 \pm 1.1$ (where a higher rating means better compliance), for non-abusers it was $3.5 \pm 0.8$, and for all patients it was $3.22 \pm 1.0$. Non-abusers had better 1-year post-discharge compliance than abusers $(t=2.9, d f=52, p<.01)$. No other rating of outcome/functioning at one year post-discharge was significantly related to substance use.

\section{Discussion}

The frequency of substance abuse among schizophrenic patients in the present investigation, $37 \%$, is similar to the rate of abuse found by other investigators (Siris et al., 1988; 
Test et al., 1989). A larger number of male as compared to female patients with substance abuse and the high frequency of polysubstance abuse is also consistent with previous studies (Bowers \& Swigar, 1983; Negrete et al., 1986; Siris et al., 1988). The percentage of patients in our sample who abused cannabis and alcohol tended to be higher than in previously published work (Miller \& Tanenbaum, 1989; Siris et al., 1988), particularly regarding cannabis. These differences may reflect differences in definition of substance abuse. Our method of classification of substance abuse was based on the impression of the evaluating psychiatrist who weighed the frequency and amount of use of each substance; this system of classification is similar to that of Breakey et al. (1974). The system of classification is not ideal as it is based on information gleaned from a chart review. Patients categorized as abusers did meet DSM III-R criteria for substance abuse; however, the chart information was not of sufficient detail to allow one to diagnose DSM III-R substance dependence for all patients in the abuser group. Nevertheless, the fact that positive urine toxicology screens and/or blood alcohol levels were significantly more prevalent in those patients classified as substance abusers than those classified as non-abusers in part validates our scheme. Those individuals who were felt to abuse substances significantly were also those who were more likely to be using these agents in the week before admission.

Similar to other investigators (Breakey et al., 1974; Mueser et al., 1990), we found that schizophrenic patients who abused substances had a younger age at first hospitalization when compared to patients in whom substance abuse was not a major factor. This result would tend to suggest a precipitating role for substance abuse in individuals predisposed to schizophrenia. However, the lack of information regarding the timing of onset of substance use relative to the onset of psychotic symptoms limits the strength of this finding. The absence of a relationship between age of first symptoms and substance abuse status is somewhat surprising, but may reflect the relative lack of reliability of this measure when compared with age of first hospitalization.

Our observation that schizophrenic patients who abuse substances of any kind were less likely to comply with treatment, thus possibly worsening their prognosis, is similar to previously published reports (Miller \& Tanenbaum, 1989). Indeed, it has been shown that dually-diagnosed patients (including patients with schizophrenia) tend to have poorer response to antipsychotic medication treatment (Bowers et al., 1990), more hospitalizations, be more often unemployed and homeless, and more likely to be violent and to become involved with the legal system (Drake \& Wallach, 1989). These findings suggest that abuse of substances may have a profound influence on short-term course of illness.

Andreasson et al. (1989) reported no difference in family history of schizophrenia between abusers and non-abusers of cannabis, yet concluded that their findings support the hypothesis that cannabis plays an etiologic role in schizophrenia. The absence of a significant relationship between substance abuse and family history of schizophrenia in our data does not allow one to draw conclusions regarding the relationship between genetic predisposition to schizophrenia and substance abuse. If substance abuse can cause schizophrenia, then substance-abusing schizophrenic patients would likely have sporadic (non-genetic) schizophrenia and should exhibit a lower family history of schizophrenia than non-substanceabusing schizophrenic patients. If substance abuse tends to precipitate schizophrenia in genetically predisposed individuals, then patients in the substance-abusing groups would 
be expected to have a higher rate of shizophrenia in family members. The relatively small number of subjects, especially those with a family history of schizophrenia, and the absence of a standardized method of data gathering regarding family history and substance abuse limit the conclusions that can be drawn from these findings.

The use of substances as self-medication has been disputed, although it is fair to assume that some patients believe their abuse is therapeutic (Test et al., 1989). The use of anticholinergic (e.g. cannabis) and/or dopaminergic agents (e.g. amphetamines) is thought to improve negative symptoms of schizophrenia, and a self-medication hypothesis would suggest that schizophrenic patients suffering from pronounced negative symptoms would tend to abuse these substances (Tandon \& Greden, 1989). However, our analysis shows no significant difference in negative symptoms between abusers and non-abusers which does not support the idea that patients were using drugs to decrease negative symptoms (i.e. anhedonia). We did find a positive association between lower pre- and post-treatment depression ratings and substance abuse in schizophrenic patients. Post-psychotic depression is a common occurrence in schizophrenia (Siris et al., 1981; Tandon \& Greden, 1989; Weiss et al., 1989), yet little is understood about its etiology. As we found no difference for negative symptoms, the HRSD scores are therefore more likely indicative of severity of depressive symptoms which at pretreatment may have been reduced by drugs of abuse, consistent with a self-medication hypothesis. It is also possible that in the substanceabusing patients non-compliance with antipsychotic medication before treatment may have decreased depressive symptoms, but this conclusion is less tenable after acute treatment as both groups received medication. However, it should be stressed that the magnitude of depressive symptoms were mild to moderate at pre-treatment, and low after treatment for both groups and that both abusers and non-abusers improved significantly on this item with treatment. The lack of significant findings with other clinical ratings (BPRS, SANS), is contrary to other work that has demonstrated an increase in positive symptoms with substance abuse (Negrete et al., 1986) precisely what one would expect in the setting of dopaminergic and anticholinergic drug use by acutely ill schizophrenic patients. This may in part be due to a lack of drug use near the time of baseline assessment.

Although our study did not involve non-schizophrenic controls, the data on pattern of abuse is of interest. We found the most abused substance to be cannabis (19), followed closely by alcohol (14), and hallucinogens (4), cocaine (3), stimulants (2), and barbiturates (1) which are abused to a lesser extent. It is interesting to note the relatively low number of heavy users of cocaine in this population with respect to cannabis and alcohol; this may reflect availability of cocaine during the years these patients were admitted. Unfortunately, with the introduction of inexpensive and easily obtained crack cocaine in the late $1980 \mathrm{~s}$, users and abusers of this illicit drug are likely to rise. The effect this rise will have on the prognosis of schizophrenic patients bears watching.

A comment is in order concerning the number of statistical tests performed and their influence on the conclusions of this study. As can be seen in the results section and in Table 3, 27 statistical tests were performed in this study. Utilizing a Bonferroni correction (Grove \& Andreasen, 1982) with alpha set at 0.1 (to balance risk of Iype I and Iype II error), a pvalue of $<.003$ is required; no finding would remain significant. The Bonferroni correction is overly conservative, particularly in light of the hypothesis-generating nature of this paper. 
Nonetheless, the possibility that the findings reported occurred by chance must be borne in mind.

The limitations of this study, i.e. its retrospective design, reliance on suboptimally reliable sources and incomplete histories, absence of precise information regarding timing of onset of substance abuse and psychotic symptoms and its lack of a non-schizophrenic control group, are important to consider. However, our findings suggest that substance abuse in schizophrenia may influence onset, clinical features, course, and outcome, and is an area worthy of further inquiry and aggressive treatment intervention.

\section{References}

American Psychiatric Association (1987). Diagnostic and statistical manual, third edition-revised. Washington, D.C.: APA Press.

Andreasen, N. C. (1981). Scale for the assessment of negative symptoms (SANS). Iowa City: University of Iowa.

Andreasson, S., Allebeck, P., Engstrom, A.. \& Rydberg, U. (1987). Cannabis and schizophrenia. A longitudinal study of Swedish conscripts. Lancet, ii, 1483-1486.

Andreasson, S., Allebeck, P., \& Rydberg, U. (1989). Schizophrenia in users and nonusers of cannabis. A longitudinal study in Stockholm County. Acta Psychiatrica Scandinatica, 79, 505-510.

Bowers, M. B., \& Swigar, M. E. (1983). Vulnerability to psychosis associated with hallucinogen use. Psichiatry' Research, 9, 91-97.

Bowers, M. B., Mazure, C. M., Nelson, J. C., \& Jatlow, P. I. (1990). Psychotogenic drug use and neuroleptic response. Schizophrenia Bulletin, 16, 81-85.

Breakey, W. R., Goodell, H., Lorenz, P. C., \& McHugh, P. R. (1974). Hallucinogenic drugs as precipitants of schizophrenia. Psychological Medicine, 4, 255--261.

Dixon, L., Haas, G., Weiden, P. J., Sweeney, J., \& Frances, A. (1991). Drug abuse in schizophrenic patients: Clinical correlations and reasons for use. American Journal of Psychiatry, 148, 224-230.

Drake, R. E. \& Wallach, M. A. (1989). Substance abuse among the chronically mentally ill. Hospital and Community Psychiatrv, 40, 1041-1046.

Feighner, J. P., Robins, E., Guze, S. B., Woodruff, R. A.. Winokur, G., \& Munoz, R. (1972). Diagnostic criteria for use in psychiatric research. Archives of General Psychiatry, 26, 57 63 .

Grove, W. M., \& Andreasen, N. C. (1982). Simultaneous tests of many hypotheses in exploratory research. Journal of Nerrous and Mental Disease, 170, 3-8.

Hamilton, M. (1960). A rating scale for depression. Journal of Neurology, Neurosurgery, and Psl'chiatry, 23, 56 62.

Johnson, B. A., Smith, B. L., \& Taylor, P. (1988). Cannabis and schizophrenia. Lancet, i, 592-593.

Knudsen, P. \& Vilmar. T. (1984). Cannabis and neuroleptic agents in schizophrenia. Acta Psychiatrica Scandinavica, 69, 162-174.

Miller, F. T., \& Tanenbaum, J. H. (1989). Drug abuse in schizophrenia. Hospital and Community Psychiatry, 40, 847849

Mueser, K. T., Yarnold, P. R., Levinson, D. F., Singh, H., Bellack, A. S., Kee, K., Morrison, R. L., \& Yadalam, K. G. (1990). Prevalence of substance abuse in schizophrenia: Demngraphic and clinical correlates. Srhizophrenia Bulletin, 16, 31-56.

Negrete, J. C., Knapp, W. P., Douglas, D. E., \& Smith, W. B. (1986). Cannabis affects the severity of schizophrenic symptoms: results of a clinical survey. Psychological Medicine, 16, 515-520.

Overall, J. E., \& Gorham, D. R. (1962). The brief psychiatric rating scale. Psychological Reports, 10, $799-812$.

Regier, D. A., Farmer, M. E., Rae, D. S., Locke, B. Z., Keith, S. J., Judd, L. L.. \& Goodwin, F. K. (1990). Comorbidity of mental disorders with alcohol and other drug abuse. Joumal of the American Medical Association, 264, 2511-2518.

Richard, M. L., Liskow, B. I., \& Perry, P. J. (1985). Recent psychostimulant use in hospitalized schizophrenics. Journal of Clinical Psychiatry, 46, 79-83.

Safer. D. J. (1987). Substance abuse by young adult chronic patients. Mospital and Community Psychiatry, 38, $511-514$

Schneier, F. R., \& Siris, S. G. (1987). A review of psychoactive substance use and abuse in schizophrenia: Patterns of drug choice. Journal of Nerrous and Mental Disease, 175, 641-652 
Siris, S. G., Harmon, G. K., \& Endicott, J. (1981). Postpsychotic depressive symptoms in hospitalized schizophrenic patients. Archites of General Psychiatry, 38, 1122-1123.

Siris, S. G., Kane, J. M., Frechen, K., Sellew, A. P., Mandeli, J., \& Fasano-Dube, B. (1988). History of substance abuse in patients with postpsychotic depressions. Comprehensive Psychiatry, 29, 550-557.

Straus, J. S., \& Carpenter, W. T. (1972). The prediction of outcome in schizophrenia. Archives of General Psychiatry, 27, 739-746.

Tandon, R., \& Greden, J. F. (1989). Cholinergic hyperactivity and negative schizophrenic symptoms. Archives of General Psychiatry, 46, 745-753.

Test, M. A., Wallisch, L. S., Allness, D. J., \& Ripp, K. (1989). Substance use in young adults with schizophrenic disorders. Schizophrenia Bulletin, 15, 465-476.

Treffert, D. A. (1978). Marijuana use in schizophrenia: A clear hazard. American Journal of Psychiatry, 135, 12131215.

Tsuang, M. T., Simpson, J. C., \& Kronfol, Z. (1982). Subtypes of drug abuse with psychosis. Archives of General Psychiatry, 39, 141-147.

Turner, W. M., \& Tsuang, M. T. (1990). Impact of substance abuse on the course and outcome of schizophrenia. Schizophrenia Bulletin, 16, 87-95.

Weiss, K. J., Valdiserri, E. V., \& Dubin, W. R. (1989). Understanding depression in schizophrenia. Hospital and Community Psychiatry, 40, 849-851.

Weller, M. P. I., Ang, P. C. Latimer-Sayer, D. T., \& Zachary, A. (1988). Drug abuse and mental illness. Lancet, ii, 997.

Zisook, S., Heaton, R., Moranville, J., Kuck, J., Jernigan. T., \& Braff, D. (1992). Past substance abuse and clinical course of schizophrenia. American Journal of Psychiatry, 149, 552-553. 